\title{
Jtem Analysis di Prove di Ascolto a Scelta Multipla della Certificazione di Jtaliano per Stranieri CILS
}

\author{
Item Analysis in Multiple-Choice Listening Tests from CILS \\ Certificate in Italian as a Foreign Language
}

Paulo Torresan*

\begin{abstract}
Il saggio si concentra sullo studio di tre prove di ascolto a scelta multipla della certificazione di italiano a stranieri CILS (livello B1, sessioni estive 2009, 2012).L'Item Analysis è la ricognizione, sulla base di dati statistici elaborati a partire dalle risposte di un campione, del comportamento di ogni singolo item. Viene data risposta a domande del tipo: per tramite degliitemsi discrimina a sufficienza tra candidati di diversa competenza? Chiavi e distrattori funzionano adeguatamente? Dalla nostra indagine emergono alcuni problemi di sottocalibrazione, di non corrispondenza tra item e informazioni presenti nel testo, di distribuzione degli item; in un caso l'item, per come è costruito, rischia di fuorviare il test taker (item \# 2, sessione estiva 2012). Lo studio, oltre ad offrire un esempio di analisi critica di un test, consente al lettore di prendere coscienza delle difficoltà che la progettazione di un'esercitazione ampiamente usata, sia in sede di testingche in sede di didattica, qual è il quesito a scelta multipla, comporta.
\end{abstract}

Parole-chiave: Italiano come lingua straniera,valutazione dell'abilità di ascolto, Item Analysis

\footnotetext{
* Doutor em Linguística e Filologia Românica pela Universidade de Veneza. Professor na Universidade de Catania. Contato: piroclastico@tin.it.
} 
Abstract: This paper analyses three multiple-choice listening tests from CILS certificate in Italian as a Foreign Language (level B1, summer session 2009 and 2012).Item Analysis involves examining the behavior of each individual item based on statistical data on answers from a sample. It offers answers to questions such as: do the items allow for sufficient discrimination between candidates of different skill levels? Do the keys and distractors work appropriately?Our investigation reveals certain issues of undercalibration, non-correspondence between items and information present in the text, and item distribution. In one case, the item's construction risks misleading the test taker (item \#2, summer session 2012). As well as providing an example of item analysis, this study allows the reader to gain awareness of how difficult it is to design an exercise widely used in both testing and teaching centers, that is, the multiple-choice question.

Key words: Italian as a FL, Assessing Listening, Item Analysis

\section{Le prove}

Le prove oggetto di analisi sono tre test a scelta multipla per valutare l'ascolto, composti ciascuno da sette item, e basati, rispettivamente, su:

- un dialogo esteso (prova di ascolto \# 2 della sessione estiva CILS 2009; cfr. \1.1.)

(prova di ascolto \# 2 della sessione estiva CILS 2012; cfr. \1.2.)

- sette microdialoghi (prova di ascolto \# 1 della sessione estiva CILS 2012; cfr. $\left.\int 1.3\right)^{1}$

\subsection{Test di ascolto a scelta multipla su dialogo esteso (sessione estiva CILS 2009)}

Il primotest di ascolto a scelta multipla su cui ci siamo concentrati riguarda un dialogo esteso (prova di ascolto \# 2 della sessione estiva CILS (2009).

${ }^{1}$ Un ringraziamento particolare a Furio Nave per l'elaborazione delle immagini 1 e 3 nonché ai colleghi sparsi per il pianeta che ci hanno aiutato nella raccolta dei dati. 


\subsubsection{Le istruzioni}

Le istruzioni risultano chiare e precise.

Ascolta il testo: è un dialogo fra due studenti. Poi completa le frasi. Scegli una delle quattro proposte di completamento che ti diamo per ogni frase.

\subsubsection{Riferimenti al CEFR}

Il testo pare sottodimensionato rispetto alle indicazioni del Quadro: gran parte degli item paiono corrispondere al livello A2 (il corsivo è nostro).

\begin{tabular}{|c|l|}
\hline & \multicolumn{1}{|c|}{ ASCOLTARE MEZZI DI COMUNICAZIONE AUDIO E } \\
REGISTRAZIONI
\end{tabular}

La velocità d'eloquio è rallentata al punto che, in alcuni tratti, lo scambio può risultare artificiale agli orecchi di un madrelingua. A detta di Canepari, l'interprete femminile è "assurda ortologicamente" (comunicazione personale, 30.12.2013), manca cioè di naturalezza.

\subsubsection{Analisi della distribuzione delle corrispondenze delle chiavi nel testo}

Si dà un doppio problema nella distribuzione degli item:

- la non progressione

- l'eccessiva concentrazione in alcune parti del testo

Indicazioni presenti nelle Guidelines for Writers of Listening Test (d'ora in poi $G W L T)^{2}$, cui molti esperti si rifanno, sono così contravvenute:

${ }^{2}$ Sono allestite dal British Council ungherese, all'interno del Progetto Into Europe, a cui si può accedere mediante il sito dell'Università di Lancaster (cfr. Sitografia). Si ispirano a Fehérváryné, Pižorn, 2005. 
"Items must follow the test sequence" (\# 2.3.2)

"Items must be spread evenly through the text" (\# 2.3.3)

Nell'immagine qui sotto (fig. 1) è rappresentata la distribuzione delle corrispondenze delle chiavi nel testo audio. Si notano:

- un vuoto consistente all'inizio (46" privi di informazioni soggette a verifica; nell'intervallo 12 "'-58”)

- un'anticipazione della risposta all'item \# 4 rispetto alle risposte agli item \#\# 2, 3

- una concentrazione di due gruppi di item: \#\# 4, 2, 3 e \#\# 5, 6
โ
$\Delta ?$
包 饮
7

$\mid \frac{17}{47}$

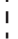

$0^{\prime \prime}$
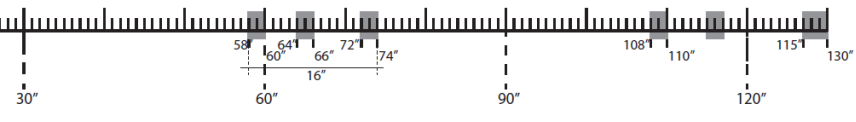

|سلس

Fig. 1. Distribuzione delle corrispondenze delle chiavi nel brano audio della prova di ascolto \# 2.

Certificazione CILS, Sessione gingno 2009, livello B1.

Qui a seguire, la distribuzione delle corrispondenze delle chiavi è evidenziata nella trascrizione del brano audio.

TRASCRIZIONE BRANO AUDIO

[MONICA]- Ciao Andrea.

[ANDREA]- Ciao Monica, come stai?

[MONICA]- Bene, (1) sono stata a visitare la scuola, un liceo, aspetta come si chiama... ah! Liceo Galilei.

[ANDREA]- Lo conosco, è un liceo scientifico.

\section{ITEM}

1. Monica è appena uscita
A) da una lezione in laboratorio.
B) dalla visita ad una nuova scuola.
C) da un ${ }^{3}$ studio medico.
D) dalla casa di un'amica.

${ }^{3}$ L'errore "*un studio medico" è presente nell’originale (si legga, ovviamente, "uno studio medico"). 
[MONICA]- Sì. La settimana scorsa sono venuti nella mia classe gli insegnati di questo liceo.

[ANDREA]- A fare l'orientamento?

[MONICA]- Sì, mi è interessato e così sono andata a vedere un po' l'ambiente. Gli insegnanti del liceo ci hanno fatto vedere le aule, i laboratori, tutte le apparecchiature tecniche.

[ANDREA]- È la prima scuola che vedi?

[MONICA]- No, ho già visto un istituto tecnico e un liceo linguistico.

[ANDREA]- E che pensi di fare?

[MONICA]- Penso di iscrivermi al Liceo Galilei. E tu a scuola hai fatto l'orientamento?

[ANDREA]- Certo, anche troppo! Sono venuti i professori di tutte le scuole superiori ed (4) io ora bo una grande confusione in testa!

[MONICA]- - Io invece no, faccio il liceo scientifico (2) e poi voglio studiare medicina.

[ANDREA]- Io non sono ancora sicuro, anche a me piacerebbe fare il liceo scientifico, (3) e poi ingegneria, indirizzo informatico, ma sono molti anni di studio. Chissà se ce la faccio!

[MONICA]- Se non ce la fai puoi sempre cercarti un lavoro.

[ANDREA]- Già. Senti, ma al liceo dove sei stata, organizzano gli esami di certificazione?

[MONICA]- Per le lingue straniere? [ANDREA]- Esatto.

\section{In futuro, Monica pensa di}

A) iscriversi alla facoltà di medicina.

B) seguire un corso di informatica.

C) studiare più a lungo possibile.

D) trovare presto un lavoro.

3. Andrea, invece, vorrebbe

A) fare l'insegnante.

B) diventare ingegnere.

C) dedicarsi alla ricerca.

D) lavorare come tecnico di laboratorio.

\section{Andrea afferma di}

A) voler fare un esame di certificazione informatica.

B) trovare poco chiari i suoi insegnanti.

C) avere le idee confuse sugli studi futuri.

D) essere molto stanco per il troppo studio.

\section{A Monica interessa molto}

A) imparare le lingue straniere.

B) studiare all'estero.

C) essere sempre preparata.

D) risparmiare sui libri.

\section{Andrea deve andare a}
A) studiare in biblioteca.
B) comprare un libro.
C) parlare con un professore.
D) seguire un corso di inglese. 
[MONICA]- Sì, fanno gli esami per l'inglese e lo spagnolo. La scuola si occupa dell'iscrizione, e c'è una riduzione sulla tassa, e poi fa anche i corsi di preparazione all'esame. Io li voglio fare tutti e due. Può essere utile e poi (5) a me piace molto studiare le lingue.

[ANDREA]- Sì. Anche a me. Ora ti devo salutare.

[MONICA]- Dove vai? [ANDREA]- (6) Vado in libreria a cercare un libro che devo leggere.

[MONICA]- Che libro?

[ANDREA]- Un romanzo che ci ha consigliato il professore di italiano. E tu dove vai?

[MONICA]- Vado a casa a studiare. (7) Devo finire il programma di storia e ripassare scienze, domani il professore mi interroga di sicuro.

\section{Monica va a casa per}

A) finire di leggere un romanzo.

B) compilare la domanda per la scuola.

C) aiutare la sua mamma.

D) prepararsi per un'interrogazione.

\subsubsection{Analisi degli item}

Abbiamo somministrato la prova a 184 studenti di livello B1, avvalendoci della disponibilità di colleghi che operano sia in Italia (in prevalenza nelle scuole private) che all'estero (in prevalenza negli Istituti di Cultura e nelle Università).

A seguire riportiamo i Facility Value (d'ora in poi FV) 4 .

Gli item \#\# 1, 2, 5 sono estremamente facili (FV > 90); in particolare l'item \# 1 pare scontato (181 studenti su 184 hanno indovinato la risposta). L'item più difficile è il \# 4, in corrispondenza del quale si registra peraltro il più alto numero di risposte mancate (6).

${ }^{4}$ Il facilityvalue (FV) corrisponde con la percentuale di studenti che rispondono correttamente ai singoli item.

Si ritiene che un item, in un proficiency test qual è il CILS, 'funzioni' nella misura in cui discrimina gli esaminandi in base alla competenza, vale a dire distingue i candidati 


\begin{tabular}{ccc}
\hline item $\#$ & FV & risposte mancate \\
\hline 1 & 98,4 & 1 \\
2 & 91,3 & 3 \\
3 & 68,5 & 2 \\
4 & 52,7 & 6 \\
5 & 96,7 & 1 \\
6 & 77,2 & 1 \\
7 & 70,1 & 1 \\
\hline
\end{tabular}

competenti da quelli che non lo sono, in riferimento al livello per il quale il test è stato confezionato. Se l'item risulta troppo facile (e quindi non discrimina a sufficienza) o al contrario se risulta troppo difficile, la prova è in disequilibrio rispetto al livello al quale viene riferita (MCNAMARA, 2000, p. 60-61): "Where the text purpose is to make distinctions between candidates, to spread them out in terms of their performance on the texts, the items should be neither too easy nor too difficult. If the items are too easy, then people with differing levels of ability or knowledge will all get them right, and the differences in ability or knowledge will not be revealed by the item. Similarly if the items are too hard, then able and less able candidates alike will get them wrong, and the item won't help us in distinguishing between them".

Quale FV considerare per stabilire l'appropriatezza di un item rispetto al livello dichiarato?

Lo stessoautoredichiara (2000, p. 67): "Ideal item facility [i.e. Facility Value, ndt.] is 0.5 [i.e. $50 \%$, ndt.] but of course it is hard to hit this target exactly, and a range of item facilities from 0.33 [i.e. 33\%, ndt.] to 0.67 [i.e. 67\%, ndt.] is usually accepted".

La posizione di Green riconoscemarginipiùampi (2013, pp. 26-27): "The most useful information about a test taker's proficiency comes from facility values which are around 50 per cent (see POPHAM2000) as this value suggests that the item might be discriminating between the test takers (though it is not always the case); 40 to 60 per cent (see BACHMAN2004), which are the values many test developers use when making initial decisions about whether an item is working or not.

Facility values between 20 and 80 per cent can also provide useful information provided the items still discriminate and contribute to the test's internal consistency [...].

Facility values of below 20 per cent and above 80 per cent in a proficiency test suggest that most of the test population is either answering the item incorrectly or correctly respectively, which reduces the chance of gaining useful information about the test takers and/or the items". 
L'istogramma (fig. 2) mostra la distribuzione dei punteggi positivi (risposte corrette) complessivi raggiunti dal campione. 66 studenti hanno risposto correttamente a 6 item su 7 . Si tratta di un profilo con disallineamento negativo (negatively skewed): il test è, cioè, piuttosto facile per il campione di candidati cui è stato somministrato.

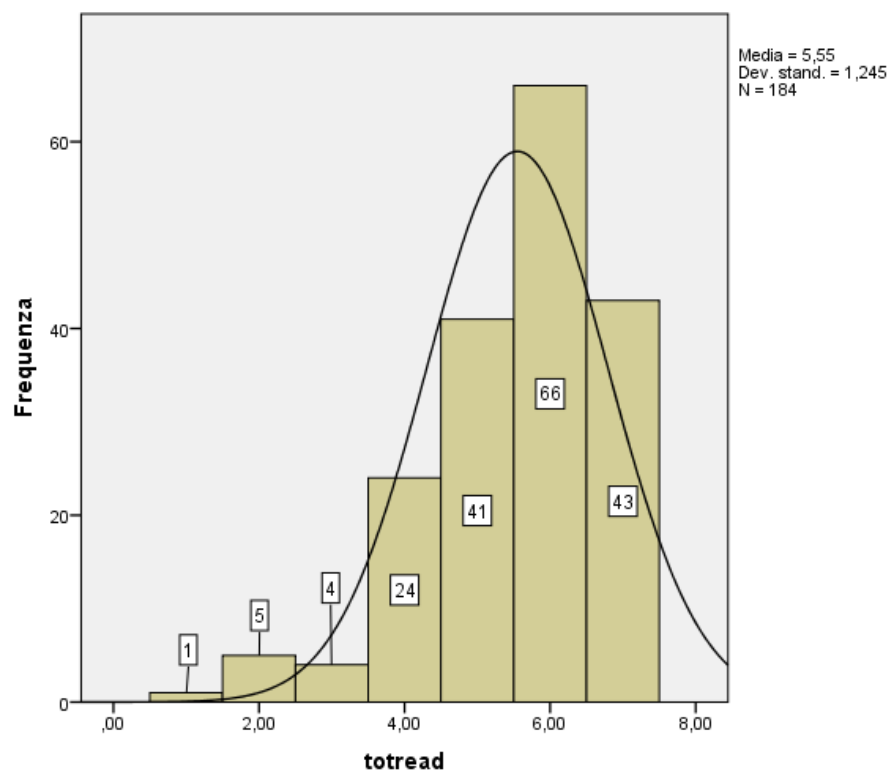

Fig. 2. Punteggi dei candidati alla prova di ascolto \# 2.

Certificazione CILS, Sessione giugno 2009, livello B1

Consideriamo le specificità di ciascun item.

item \# 1

È un item scontato. Il distrattore a) discrimina pochissimo; i distrattori c) e d) non sono stati scelti da nessuno. 


\section{Monica è appena uscita}
A) da una lezione in laboratorio.
B) dalla visita ad una nuova scuola.
C) da un ${ }^{5}$ studio medico.
D) dalla casa di un'amica.

\begin{tabular}{|c|c|c|c|c|c|}
\hline & & Frequenza & Percentuale & $\begin{array}{c}\text { Percentuale } \\
\text { valida }^{6}\end{array}$ & $\begin{array}{l}\text { Percentuale } \\
\text { cumulata }^{7}\end{array}$ \\
\hline \multirow{3}{*}{ Validi } & $\mathrm{A}$ & 2 & 1,1 & 1,1 & 1,1 \\
\hline & $\mathrm{B}=$ Chiave & 181 & 98,4 & 98,9 & 100,0 \\
\hline & Totale & 183 & 99,5 & 100,0 & \\
\hline \multirow{2}{*}{$\begin{array}{l}\text { Mancanti } \\
\text { Totale }\end{array}$} & Nessuna risposta & 1 & 0,5 & & \\
\hline & & 184 & 100,0 & & \\
\hline
\end{tabular}

\section{item \# 2}

Item molto facile; i distrattori non discriminano.
2. In futuro, Monica pensa di
A) iscriversi alla facoltà di medicina.
B) seguire un corso di informatica.
C) studiare più a lungo possibile.
D) trovare presto un lavoro.

\begin{tabular}{llrrrr}
\hline & Frequenza & Percentuale & $\begin{array}{c}\text { Percentuale } \\
\text { valida }\end{array}$ & $\begin{array}{c}\text { Percentuale } \\
\text { cumulata }\end{array}$ \\
\hline \multirow{6}{*}{ Validi } & A=Chiave & 168 & 91,3 & 92,3 & 92,3 \\
& $\mathrm{~B}$ & 6 & 3,3 & 3,3 & 95,6 \\
& $\mathrm{C}$ & 6 & 3,3 & 3,3 & 98,9 \\
& $\mathrm{D}$ & 1 & 0,5 & 0,5 & 99,5 \\
Mancanti & Totale & 181 & 98,4 & 100,0 & \\
Totale & Nessuna risposta & 3 & 1,6 & & \\
\hline
\end{tabular}

5 Si veda la nota a piè di pagina n. 3 .

${ }^{6}$ Percentuale valida: depurata delle mancate risposte.

7 Percentuale cumulata: si tratta della somma tra la singola percentuale e quelle che precedono. 
L'item pare essere adeguatamente calibrato.

\begin{tabular}{|c|c|c|c|c|c|}
\hline & & $\begin{array}{l}\text { 3. Andrea, invec } \\
\text { A) fare l'insegnan } \\
\text { B) diventare inge } \\
\text { C) dedicarsi alla } \\
\text { D) lavorare come } \\
\text { laboratorio. }\end{array}$ & $\begin{array}{l}\text {, vorrebbe } \\
\text { e. } \\
\text { ere. } \\
\text { erca. } \\
\text { ecnico di }\end{array}$ & & \\
\hline & & Frequenza & Percentuale & $\begin{array}{c}\text { Percentuale } \\
\text { valida }\end{array}$ & $\begin{array}{c}\text { Percentuale } \\
\text { cumulata }\end{array}$ \\
\hline & A & 25 & 13,6 & 13,7 & 13,7 \\
\hline & $\mathrm{B}=$ Chiave & 126 & 68,5 & 69,2 & 83,0 \\
\hline Validi & C & 13 & 7,1 & 7,1 & 90,1 \\
\hline & D & 18 & 9,8 & 9,9 & 100,0 \\
\hline & Totale & 182 & 98,9 & 100,0 & \\
\hline Mancanti & Nessuna rispc & sta & 1,1 & & \\
\hline Totale & & 184 & 100,0 & & \\
\hline
\end{tabular}

item \# 4

L'item si è rivelato arduo per il campione cui è stato somministrato.

Se sommiamo la percentuale di test taker che hanno optato per distrattore a) con quella delle risposte mancanti, raggiungiamo la soglia del $40 \%$ di opzioni inesatte!

La spiegazione di questa complessità può risiedere, a nostro giudizio, in un problema di distribuzione delle corrispondenze delle chiavi nel testo. La risposta all'item \# 4, come abbiamo visto al $\int 1.1 .3$.), è presente in una porzione di testo che precede la porzione di testo nella quale sono contenute le risposte agli item \#\# 2 e 3. Vien da dedurre che alcuni studenti abbiano atteso (invano) una risposta nella porzione di testo 'errata', dove cioè è 'naturale' pensare essa si trovi.

Oltretutto, l'attrattività del distrattore a) ("[Andrea afferma di] voler fare un esame di certificazione informatica") si spiega per il fatto che, nel testo, il parlante dice di voler frequentare "ingegneria, indirizzo informatico"; ci pare che chi non abbia familiarità con il mondo dell'università possa interpretare "indirizzo informatico" come il corrispettivo di "certificazione informatica". Si tratta di una congettura che andrebbe, comunque, verificata. 


\section{Andrea afferma di}

A) voler fare un esame di certificazione informatica.

B) trovare poco chiari i suoi insegnanti.

C) avere le idee confuse sugli studi futuri.

D) essere molto stanco per il troppo studio.

\begin{tabular}{llrrrr}
\hline & Frequenza & Percentuale & $\begin{array}{c}\text { Percentuale } \\
\text { valida }\end{array}$ & $\begin{array}{c}\text { Percentuale } \\
\text { cumulata }\end{array}$ \\
\hline \multirow{6}{*}{ Validi } & A & 67 & 36,4 & 37,4 & 37,4 \\
& $\mathrm{~B}$ & 7 & 3,8 & 3,9 & 41,3 \\
& $\mathrm{C}=$ Chiave & 97 & 52,7 & 54,2 & 95,5 \\
& $\mathrm{D}$ & 7 & 3,8 & 3,9 & 99,4 \\
& $\mathrm{M}$ & 1 & 0,5 & 0,6 & 100,0 \\
Mancanti & Totale & 179 & 97,3 & 100,0 & \\
Totale & Nessuna risposta & 5 & 2,7 & & \\
\hline
\end{tabular}

item \# 5

L'item si rivela scontato. La corrispondenza chiave-testo è evidente: " $a$ me piace molto studiare le lingue" (testo); "a Monica interessa molto imparare le lingue straniere" (chiave a). I distrattori non sono efficaci.
5. A Monica interessa molto
A) imparare le lingue straniere.
B) studiare all'estero.
C) essere sempre preparata.
D) risparmiare sui libri.

\begin{tabular}{llrrrr}
\hline & Frequenza & Percentuale & $\begin{array}{c}\text { Percentuale } \\
\text { valida }\end{array}$ & $\begin{array}{c}\text { Percentuale } \\
\text { cumulata }\end{array}$ \\
\hline \multirow{6}{*}{ Validi } & A=Chiave & 178 & 96,7 & 97,3 & 97,3 \\
& B & 2 & 1,1 & 1,1 & 98,4 \\
& $\mathrm{C}$ & 2 & 1,1 & 1,1 & 99,5 \\
& D & 1 & 0,5 & 0,5 & 100,0 \\
Mancanti & Totale & 183 & 99,5 & 100,0 & \\
Totale & Nessuna risposta & 1 & 0,5 & & \\
\hline
\end{tabular}




\section{item \# 6}

L'item ha un FV appena al di sotto della soglia prevista da Green (80): è relativamente facile.

Il distrattore a) ha attirato, comunque, un discreto numero di consensi. Nella scelta del distrattore può aver influito, nel caso degli studenti anglofoni, la presenza di un falso amico (library/biblioteca).
6. Andrea deve andare a
A) studiare in biblioteca.
B) comprare un libro.
C) parlare con un professore.
D) seguire un corso di inglese.

\begin{tabular}{llrrrr}
\hline & & Frequenza & Percentuale & $\begin{array}{c}\text { Percentuale } \\
\text { valida }\end{array}$ & $\begin{array}{c}\text { Percentuale } \\
\text { cumulata }\end{array}$ \\
\hline \multirow{4}{*}{ Validi } & A & 34 & 18,5 & 18,6 & 18,6 \\
& B=Chiave & 142 & 77,2 & 77,6 & 96,2 \\
& C & 2 & 1,1 & 1,1 & 97,3 \\
& D & 5 & 2,7 & 2,7 & 100,0 \\
Mancanti & Totale & 183 & 99,5 & 100,0 & \\
Totale & Nessuna risposta & 1 & 0,5 & & \\
\hline
\end{tabular}

item \# 7

L'item discrimina a dovere ( $\mathrm{FV}=70)$.

Il distrattore b) attira un considerevole numero di consensi $(22 \%)$. Il distrattore c), al contrario, è stato scelto da pochissimi.
7. Monica va a casa per
A) finire di leggere un romanzo.
B) compilare la domanda per la scuola.
C) aiutare la sua mamma.
D) prepararsi per un'interrogazione 


\begin{tabular}{llrrrr}
\hline & & Frequenza & Percentuale & $\begin{array}{c}\text { Percentuale } \\
\text { valida }\end{array}$ & $\begin{array}{c}\text { Percentuale } \\
\text { cumulata }\end{array}$ \\
\hline \multirow{6}{*}{ Validi } & A & 11 & 6,0 & 6,0 & 6,0 \\
& B & 41 & 22,3 & 22,4 & 28,4 \\
& C & 2 & 1,1 & 1,1 & 29,5 \\
& D=Chiave & 129 & 70,1 & 70,5 & 100,0 \\
Mancanti & Totale & 183 & 99,5 & 100,0 & \\
Totale & Nessuna risposta & 1 & 0,5 & & \\
\hline
\end{tabular}

\subsubsection{Bilancio}

Il testo audio presenta una lentezza al punto da dare l'impressione che i parlanti interagiscano in maniera meccanica e innaturale.

A compensare la relativa facilità di testo e item, vi sono caratteristiche della distribuzione degli item che rendono arduo l'ascolto: l'eccessiva concentrazione di gruppi di itemin corrispondenza di alcune parti del testo e il mancato rispetto della sequenza delle informazioni presenti nel testo.

Il fatto quindi che la prova consenta di produrre inferenze attendibili sulla capacità di comprensione orale da parte di un candidato di livello B1 è, per questi motivi, messo in discussione.

\subsection{Test di ascolto a scelta multipla su dialogo esteso (sessione estiva}

\section{CILS 2012)}

Il secondo test di ascolto oggetto di indagine riguarda, ancora una volta, un dialogo esteso, sempre per apprendenti di livello B1.

I protagonisti sono due amici che si mettono d'accordo per organizzare un'uscita il sabato sera (prova di ascolto \# 2 della sessione estiva CILS 2012).

\subsubsection{Le istruzioni}

Le istruzioni risultano chiare e precise.

Ascolta il testo: è un dialogo fra due amici.

Poi completa le frasi. Scegli una delle

quattro proposte di completamento. 


\subsubsection{Riferimenti al CEFR}

La velocità d'eloquio e il lessico paiono adeguati per il livello per il quale la prova è stata confezionata.

Lo stesso dicasi per quanto riguarda la quasi totalità degli item (nell'estratto riportiamo anche il descrittore relativo al B1 potenziato).

\begin{tabular}{|c|l|}
\hline \multirow{3}{*}{$\mathrm{B} 1+$} & \begin{tabular}{l} 
COMPRENSIONE ORALE GENERALE \\
\hline È in grado di comprendere informazioni fattuali chiare su \\
argomenti comuni relativi alla vita di tutti i giorni o al lavoro, \\
riconoscendo sia il significato generale sia le informazioni \\
specifiche, purché il discorso sia pronunciato con chiarezza in \\
un accento piuttosto familiare
\end{tabular} \\
\hline B1 & $\begin{array}{l}\text { È in grado di comprendere i punti salienti di un discorso } \\
\text { chiaro in lingua standard che tratti argomenti familiari } \\
\text { affrontati abitualmente sul lavoro, a scuola, nel tempo libero, } \\
\text { ecc., compresi dei brevi racconti. }\end{array}$ \\
\hline
\end{tabular}

Un'eccezione è costituita, tuttavia, dai primi due item: l'informazione ricercata si distacca dal testo, è marcata ed evidente. C'è da chiedersi se, a tal proposito, non siano più idonei i descrittori del livello A2 per rappresentare le strategie di cui l'allievo deve dar prova.

\begin{tabular}{|l|l|}
\hline & \multicolumn{1}{|c|}{$\begin{array}{c}\text { ASCOLTARE MEZZI DI COMUNICAZIONE } \\
\text { AUDIO E REGISTRAZIONI }\end{array}$} \\
\hline \multirow{3}{*}{ A2 } & $\begin{array}{l}\text { È in grado di comprendere ed estrare l'informazione } \\
\text { essenziale da brevi testi registrati, che trattino di argomenti } \\
\text { prevedibili di uso quotidiano e che siano pronunciati } \\
\text { lentamente e chiaramente. }\end{array}$ \\
\hline
\end{tabular}




\subsubsection{Analisi della distribuzione delle corrispondenze delle chiavi nel testo}

Si riscontra un'eccessiva concentrazione delle corrispondenze degli item in alcune parti del testo; ci sono un paio di contiguità (corrispondenze delle chiavi degli item\#\# 2-3; 4-5) e, in un caso, le corrispondenze sono incorporate l'una nell'altra (la corrispondenza della chiave dell'item \# 3 è incorporatanella corrispondenza della chiave dell'item\# 2). Riteniamo ciò possa generare confusione nel testtaker, il quale si trova, nell'attimo in cui è concentrato a rispondere a un item, ad avere difficoltà a stare al passo con l'ascolto del testo che segue (cf. NOVELLO 2014).

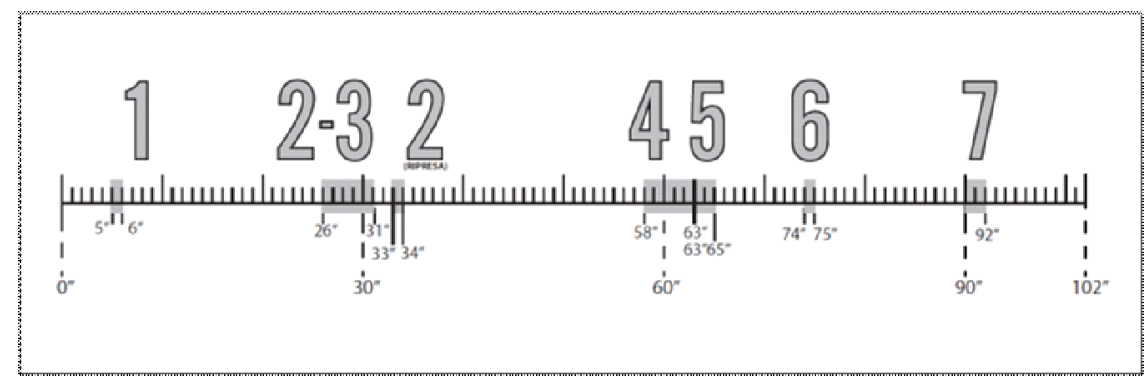

Fig. 3. Distribuzione delle corrispondenze delle chiavinel brano audio della prova di ascolto \# 2.

Certificazione CILS, Sessione gingno 2012, livello B1

Nella trascrizione del brano audio, la distribuzione delle corrispondenze è numerata ed evidenziata in corsivo. 
[PATRIZIA]- Ciao Alessandro, come stai? Sono felice di incontrarti.

[ALESSANDRO]- Sto bene, grazie. Fa piacere anche a me averti incontrata così, (1)per strada. Volevo proprio parlare con te; pensavo di telefonarti stasera.

[PATRIZIA]- Perché volevi telefonarmi? C'è qualche novità?

[ALESSANDRO]- No, niente di particolare. Volevo solo chiederti se ti va di andare insieme a "Identità golose", che c'è a Milano la prossima settimana, nel fine settimana.

[PATRIZIA]- Scusa, ma che cosa è "Identità golose"?

[ALESSANDRO]- (2) $\grave{E}$ un congresso([2/3]) internazionale di cuochi:(3) c'è l'eccellenza della cucina italiana e del mondo.

[PATRIZIA]- Ma che cosa ci facciamo noi a ([2]) un congresso di cuochi? È una noia terribile! Ti immagini sentire discussioni infinite sul carciofo DOP della Sardegna o sul cappero di Pantelleria!

[ALESSANDRO]- Ma no, non voglio portarti al convegno! La sera, però, ci sono tante iniziative interessanti ...

\section{Patrizia e Alessandro sono}

A) per strada.

B) al telefono.

C) al ristorante.

D) sull'autobus.

2. Alessandro parla a Patrizia di "Identità golose" che è
A) una scuola di cucina.
B) un nuovo ristorante.
C) un congresso di cuochi.
D) un mercato di prodotti tipici.

3. A "Identità golose" partecipano

A) cuochi di livello internazionale.

B) personaggi famosi che amano cucinare.

C) studenti di una scuola per cuochi.

D) persone comuni che vogliono diventare cuochi.

\section{Alessandro spiega a Patrizia che la sera a "Identità golose" è possibile}

A) comprare cibi pronti per cenare a casa.
B) cenare a prezzi bassi.
C) cucinare insieme a cuochi famosi.
D) assistere a spettacoli teatrali.

\section{Per andare una sera a "Identità} golose" è necessario
A) fare un'iscrizione.
B) presentarsi direttamente.
C) avere un invito.
D) prenotare in anticipo. 
[PATRIZIA]- Spiegami meglio... [ALESSANDRO]- I cuochi si esibiscono in dimostrazioni culinarie e poi i visitatori possono fare assaggi ed (4)è possibile anche cenare. Ho letto nel programma che ogni sera c'è un ristorante famoso, ma i prezzi sono contenuti, (5) però è necessario prenotare... Che ne dici, ci andiamo?

[PATRIZIA]- Sì, mi piace quest'idea. Fammi vedere se sono libera il prossimo fine settimana.

6) Potrebbe andare bene domenica sera... venerdì devo lavorare fino a tardi e sabato ho promesso ai miei genitori di andare a casa loro.

[ALESSANDRO]- Va bene, allora prenoto per domenica sera.

[PATRIZIA]- D'accordo. Ma dov'è questo "Identità golose"?

[ALESSANDRO]- È al Centro Congressi in via Gattamelata, ma non ti preoccupare, (7) passo io a prenderti. Va bene alle 8 ?

[PATRIZIA]- Sì certamente. Comunque sentiamoci per telefono qualche giorno prima.

[ALESSANDRO]- Allora ciao, a domenica.

\section{Quando Alessandro propone a Patrizia di uscire, lei dice che}

A) è fuori per lavoro nel fine settimana.

B) preferisce far tardi il sabato sera.

C) è libera solo la domenica sera.

D) le va bene qualunque giorno del fine settimana.

\section{Alessandro suggerisce a Patrizia}

A) di raggiungere a piedi "Identità golose".

B) di incontrarsi a casa di Patrizia.

C) di portare anche i suoi genitori a "Identità golose".

D) di telefonargli se cambia idea.

\subsubsection{Analisi degli item}

Abbiamo somministrato la prova a 143 studenti di livello B1, avvalendoci della disponibilità di colleghi che operano sia in Italia (in prevalenza nelle scuole private) che all'estero (in prevalenza negli Istituti di Cultura e nelle Università).

A seguire riportiamo gli $\mathrm{FV}$. 
Si noti la presenza consistente di item (\#\# 1,2,6) sottostimati rispetto alla competenza del campione.

\begin{tabular}{cccc}
\hline item\# & $\mathbf{F V}$ & risposte mancate & risposte doppie \\
\hline 1 & 96,5 & 0 & 0 \\
2 & 99,3 & 0 & 0 \\
3 & 80,4 & 2 & 0 \\
4 & 62,2 & 2 & 0 \\
5 & 79,7 & 1 & 0 \\
6 & 93,7 & 0 & 0 \\
7 & 72,7 & 2 & 1 \\
\hline
\end{tabular}

L'istogramma (fig. 4) mostra la distribuzione dei punteggi positivi (risposte corrette) complessivi raggiunti dal campione. La maggior parte degli studenti cui il test è stato somministrato (50) ha risposto correttamente a 7item su 7. Si tratta di un profilo con un evidente disallineamento negativo (negatively skewed): il test è, cioè, molto facile per il campione di candidati a cui è stato somministrato. 


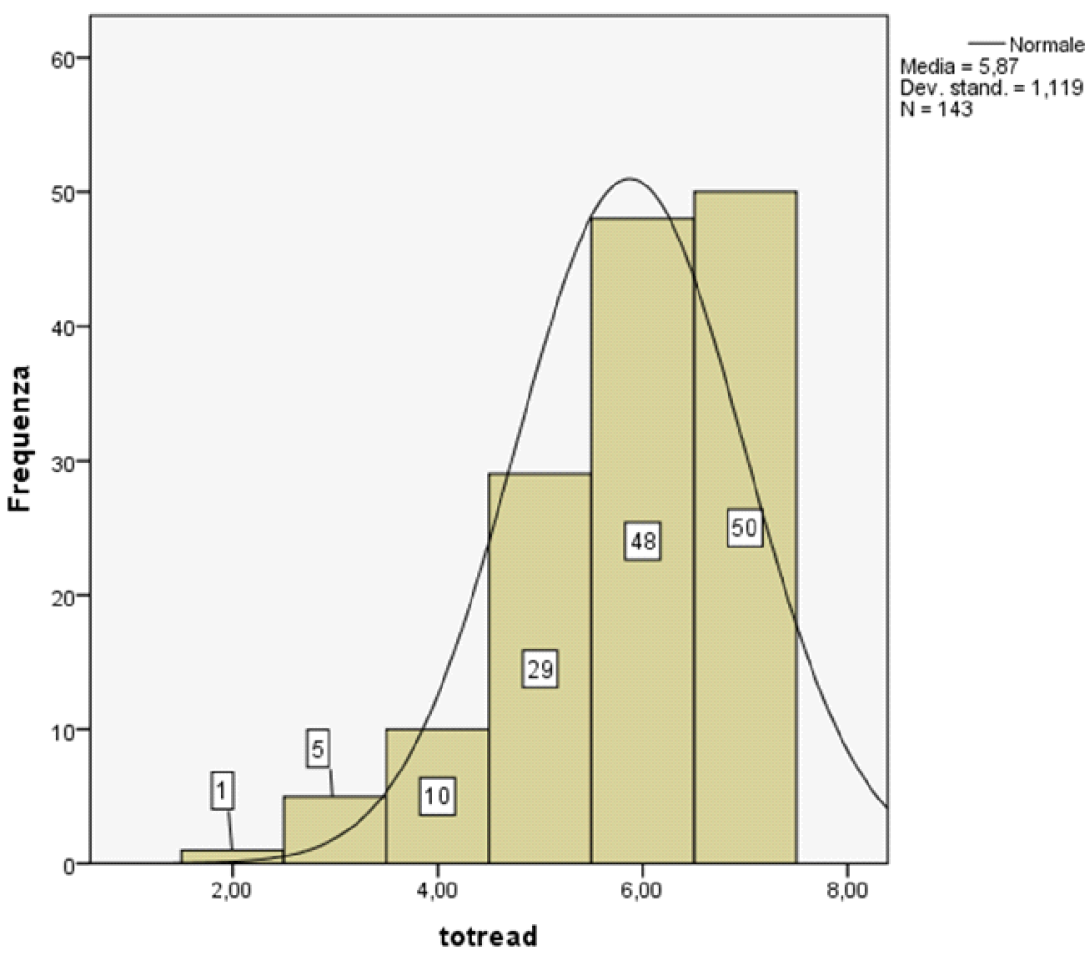

Fig. 4. Punteggi dei candidati alla prova di ascolto \# 2.

Certificazione CILS, Sessione gingno 2012, livello B1

Consideriamo le specificità di ciascun item.

item \# 1

È un item scontato: il 96,5\% degli studenti ha indovinato la risposta. L'informazione cui rimanda la chiave, infatti, si 'distacca', rispetto al flusso del discorso, con una certa evidenza, isolata da due brevissime pause.

Patrizia e Alessandro sono

A) per strada.

B) al telefono.

C) al ristorante.

D) sull'autobus. 


\begin{tabular}{rlrrrr}
\hline & Frequenza & Percentuale & $\begin{array}{c}\text { Percentuale } \\
\text { valida }\end{array}$ & $\begin{array}{c}\text { Percentuale } \\
\text { cumulata }\end{array}$ \\
\hline \multirow{3}{*}{ Validi } & A=Chiave & 138 & 96,5 & 96,5 & 96,5 \\
& B & 5 & 3,5 & 3,5 & 100,0 \\
& Totale & 143 & 100,0 & 100,0 & \\
\hline
\end{tabular}

item \# 2

L'item èovvio: la quasi totalità del campione (eccetto uno studente) ha risposto correttamente; i distrattori si rivelano inefficaci.

2. Alessandro parla a Patrizia di "Identità golose" che è

A) una scuola di cucina.

B) un nuovo ristorante.

C) un congresso di cuochi

D) un mercato di prodotti tipici.

\begin{tabular}{rlrrrr}
\hline & Frequenza & Percentuale & $\begin{array}{c}\text { Percentuale } \\
\text { valida }\end{array}$ & $\begin{array}{c}\text { Percentuale } \\
\text { cumulata }\end{array}$ \\
\hline \multirow{3}{*}{ Validi } & B & 1 & 0,7 & 0,7 &, 7 \\
& C=Chiave & 142 & 99,3 & 99,3 & 100,0 \\
& Totale & 143 & 100,0 & 100,0 & \\
\hline
\end{tabular}

\section{item \# 3}

L'item si presenta come relativamente facile. Un certo grado di attrazione l'ha esercitato il distrattore d), forse per via di un'inferenza circa il possibile ruolo di Alessandro e Patrizia al congresso. Alla scelta errata, in questo caso, può aver contribuito pure il fatto che il rimando della chiave è compresso nella porzione di testo che risponde anche all'item \# 2, come abbiamo visto al $\int 1.2 .3$.

\section{A "Identità golose" partecipano}

A) cuochi di livello internazionale.

B) personaggi famosi che amano cucinare.

C) studenti di una scuola per cuochi.

D) persone comuni che vogliono diventare cuochi. 


\begin{tabular}{llrrrr}
\hline & & Frequenza & Percentuale & $\begin{array}{c}\text { Percentuale } \\
\text { valida }\end{array}$ & $\begin{array}{c}\text { Percentuale } \\
\text { cumulata }\end{array}$ \\
\hline \multirow{6}{*}{ Validi } & A=Chiave & 115 & 80,4 & 81,6 & 81,6 \\
& $\mathrm{~B}$ & 8 & 5,6 & 5,7 & 87,2 \\
& $\mathrm{C}$ & 3 & 2,1 & 2,1 & 89,4 \\
& $\mathrm{D}$ & 15 & 10,5 & 10,6 & 100,0 \\
Mancanti & Totale & 141 & 98,6 & 100,0 & \\
Totale & Nessuna risposta & 2 & 1,4 & & \\
\hline
\end{tabular}

item \# 4

Si tratta dell'item più impegnativo (FV 62,2).

Si può supporre che l'enunciato presente nell'audio " $i$ prezzi sono contenuti" (chiave b) non sia chiaro a un apprendente di livello B1.

Ci rimane, comunque, di difficile spiegazione la notevole attrattività del distrattore c), che ha raccolto quasi il 30\% dei consensi (si tratta forse di un'inferenzaerrata del termine "assagg $\imath$ " nell'enunciato del brano audio " $i$ visitatoripossono fare assaggi"?).
4. Alessandro spiega a Patrizia che la sera a "Identità golose" è possibile
A) comprare cibi pronti per cenare a casa.
B) cenare a prezzi bassi
C) cucinare insieme a cuochi famosi.
D) assistere a spettacoli teatrali.

\begin{tabular}{llrrrr}
\hline & & Frequenza & Percentuale & $\begin{array}{r}\text { Percentuale } \\
\text { valida }\end{array}$ & $\begin{array}{c}\text { Percentuale } \\
\text { cumulata }\end{array}$ \\
\hline \multirow{6}{*}{ Validi } & A & 9 & 6,3 & 6,4 & 6,4 \\
& $\mathrm{~B}=$ Chiave & 89 & 62,2 & 63,1 & 69,5 \\
& $\mathrm{C}$ & 40 & 28,0 & 28,4 & 97,9 \\
& $\mathrm{D}$ & 3 & 2,1 & 2,1 & 100,0 \\
Mancanti & Totale & 141 & 98,6 & 100,0 & \\
Totale & Nessuna risposta & 2 & 1,4 & & \\
\hline
\end{tabular}




\section{item \# 5}

L'item è relativamente facile; il verbo "prenotare" presente nella chiave si ripete tale e quale nel dialogo (per quanto il fatto di dover prenotare "in anticipo", come recita la chiave, non sia immediatamente desumibile dal testo).
5. Per andare una serc
A) fare un'iscrizione.
B) presentarsi direttamente.
C) avere un invito.
D) prenotare in anticipo

\begin{tabular}{llrrrr}
\hline & & Frequenza & Percentuale & $\begin{array}{c}\text { Percentuale } \\
\text { valida }\end{array}$ & $\begin{array}{c}\text { Percentuale } \\
\text { cumulata }\end{array}$ \\
\hline \multirow{6}{*}{ Validi } & A & 6 & 4,2 & 4,2 & 4,2 \\
& $\mathrm{~B}$ & 8 & 5,6 & 5,6 & 9,9 \\
& $\mathrm{C}$ & 14 & 9,8 & 9,9 & 19,7 \\
& D=Chiave & 114 & 79,7 & 80,3 & 100,0 \\
& Totale & 142 & 99,3 & 100,0 & \\
Mancanti & Nessuna risposta & 1 & 0,7 & & \\
\hline
\end{tabular}

item \# 6

L'item è molto facile. L'informazione corrispondente alla chiave è espressa dall'interprete femminile in modo esplicito.
A) è fuori per lavoro nel fine settimana.
B) preferisce far tardi il sabato sera.
C) è libera solo la somenica sera.
D) le va bene qualunque giorno del fine settimana.

6. Quando Alessandro propone a Patrizia di uscire, lei dice che

\begin{tabular}{llrrrr}
\hline & Frequenza & Percentuale & $\begin{array}{c}\text { Percentuale } \\
\text { valida }\end{array}$ & $\begin{array}{c}\text { Percentuale } \\
\text { cumulata }\end{array}$ \\
\hline \multirow{5}{*}{ Validi } & A & 4 & 2,8 & 2,8 & 2,8 \\
& B & 1 & 0,7 & 0,7 & 3,5 \\
& C=Chiave & 134 & 93,7 & 93,7 & 97,2 \\
& D & 4 & 2,8 & 2,8 & 100,0 \\
& Totale & 143 & 100,0 & 100,0 & \\
\hline
\end{tabular}


Benché una discreta percentuale di test taker abbia risposto convenientemente (FV=72,7), siamo convinti che l'equivalenza tra la chiave ("incontrarsi a casa di Patrizia") e il rimando nel testo ("passo io a prenderti") possa non essere trasparente a un apprendente di livello B1 (oltretutto il luogo in cui Patrizia vive può non essere necessariamente la sua casa).
7. Alessandro suggerisce a Patrizia
A) di raggiungere a piedi "Identità golose".
B) di incontrarsi a cada di Patrizia.
C) di portare anche i suoi genitori a "Identità golose".
D) di telefonargli se cambia idea.

\begin{tabular}{llrrrr}
\hline & & Frequenza & Percentuale & $\begin{array}{c}\text { Percentuale } \\
\text { valida }\end{array}$ & $\begin{array}{c}\text { Percentuale } \\
\text { cumulata }\end{array}$ \\
\hline \multirow{6}{*}{ Validi } & A & 4 & 2,8 & 2,9 & 2,9 \\
& B $=$ Chiave & 104 & 72,7 & 74,3 & 77,1 \\
& $\mathrm{C}$ & 3 & 2,1 & 2,1 & 79,3 \\
& $\mathrm{D}$ & 29 & 20,3 & 20,7 & 100,0 \\
& Totale & 140 & 97,9 & 100,0 & \\
\multirow{5}{*}{ Mancanti } & Nessuna risposta & 2 & 1,4 & & \\
\multirow{2}{*}{ Totale } & Doppia risposta & 1 & 0,7 & & \\
& Totale & 3 & 2,1 & & \\
\hline
\end{tabular}

\subsubsection{Bilancio}

Molti itemrisultano sottodimensionati; i distrattorispesso non risultano efficaci. La prova ci pare debba essere soggetta a revisione.

\subsection{Test di ascolto a scelta multipla basato su microdialoghi (sessione estiva CILS 2012)}

L'ultimo test di ascolto oggetto di indagine riguarda sette microdialoghi, a ciascuno dei quali corrisponde un item specifico. Il livello di riferimento è sempre B1 (prova di ascolto \# 1 della sessione estiva CILS 2012).

\subsubsection{Le istruzioni}

Le istruzioni risultano chiare e precise. 
Ascolta i testi. Poi completa le frasi. Scegli una della quattro proposte di completamento.

\subsubsection{Riferimenti al CEFR}

La velocità d'eloquio pare adeguata al livello (nell'estratto riportiamo anche il descrittore relativo al B1 potenziato): non è così rallentata da suonare artificiale agli orecchi di un madrelinguacome nella prova del 2009, se non a tratti (la voce femminile nel dialogo corrispondente all'item \# 2 compie pause innaturali) ${ }^{8}$.

\begin{tabular}{|c|l|}
\hline COMPRENSIONE ORALE GENERALE \\
\hline B1+ & $\begin{array}{l}\text { È in grado di comprendere informazioni fattuali chiare su } \\
\text { argomenti comuni relativi alla vita di tutti i giorni o al lavoro, } \\
\text { riconoscendo sia il significato generale sia le informazioni } \\
\text { specifiche, purché il discorso sia pronunciato con chiarezza in un } \\
\text { accento piuttosto familiare. }\end{array}$ \\
\hline B1 & $\begin{array}{l}\text { È in grado di comprendere i punti salienti di un discorso chiaro in } \\
\text { lingua standard che tratti argomenti familiari affrontati } \\
\text { abitualmente sul lavoro, a scuola, nel tempo libero, ecc., } \\
\text { compresi dei brevi racconti. }\end{array}$ \\
\hline
\end{tabular}

Un'eccezione è costituita da un solo item (\# 3), dove l'inferenza richiesta non riguarda il significato di una parola (B1) quanto piuttosto una rete di significati sulla base di indizi contestuali (B2).

${ }^{8}$ Sarebbe da valutare, comunque, l'opportunità di avere voci distinte per ogni dialogo, dal momento che i personaggi coinvolti sono di volta in volta diversi. 


\begin{tabular}{|c|l|}
\hline \multirow{3}{*}{ B2 } & \multicolumn{1}{|c|}{ INDIVIDUARE INDIZI E FARE INFERENZE } \\
\hline \multirow{3}{*}{ B1 } & $\begin{array}{l}\text { È in grado di comprendere informazioni fattuali chiare su } \\
\text { argomenti comuni relativi alla vita di tutti i giorni o al lavoro, } \\
\text { riconoscendo sia il significato generale sia le informazioni } \\
\text { specifiche, purché il discorso sia pronunciato con chiarezza in } \\
\text { un accento piuttosto familiare. }\end{array}$ \\
\hline $\begin{array}{l}\text { È in grado di identificare in base al contesto parole } \\
\text { sconosciute, relativamente ad argomenti che si riferiscono al } \\
\text { suo campo di interesse. } \\
\text { È in grado di estrapolare dal contesto il significato di una } \\
\text { parola sconosciuta e ricostruire il significato della frase, a } \\
\text { condizione di avere familiarità con l'argomento in questione. }\end{array}$ \\
\hline
\end{tabular}

\subsubsection{Analisi degli item}

Abbiamo somministrato la prova a 140 studenti di livello B1, avvalendoci della disponibilità di colleghi che operano sia in Italia (in prevalenza nelle scuole private) che all'estero (in prevalenza negli Istituti di Cultura e nelle università).

A seguire riportiamo gli FV. Colpisce il fatto che gli item paiono meglio calibrati rispetto alle prove precedenti; non abbiamo FV al di sopra del 90. L'item \# 3 si distacca dagli altri per un valore basso: merita un'analisi più dettagliata. Si evidenzia anche un numero considerevole di risposte invalide (mancate e doppie) in corrispondenza all'item \# 2.

\begin{tabular}{cccc}
\hline item\# & FV & risposte mancate & risposte doppie \\
\hline 1 & 84,3 & 3 & 0 \\
2 & 72,1 & 5 & 1 \\
3 & 60,0 & 1 & 0 \\
4 & 80,7 & 0 & 3 \\
5 & 85,0 & 1 & 0 \\
6 & 84,3 & 0 & 1 \\
\hline 7 & 81,4 & 3 & 0 \\
\hline
\end{tabular}


L'istogramma (fig. 5) mostra la distribuzione dei punteggi positivi (risposte corrette) complessivi raggiunti dal campione. La maggior parte degli studenti cui il test è stato somministrato (48) ha risposto correttamente a Gitem su 7. Si tratta di un profilo con disallineamento negativo (negatively skewed): il test è, cioè, piuttosto facile per il campione di candidati a cui è stato somministrato.

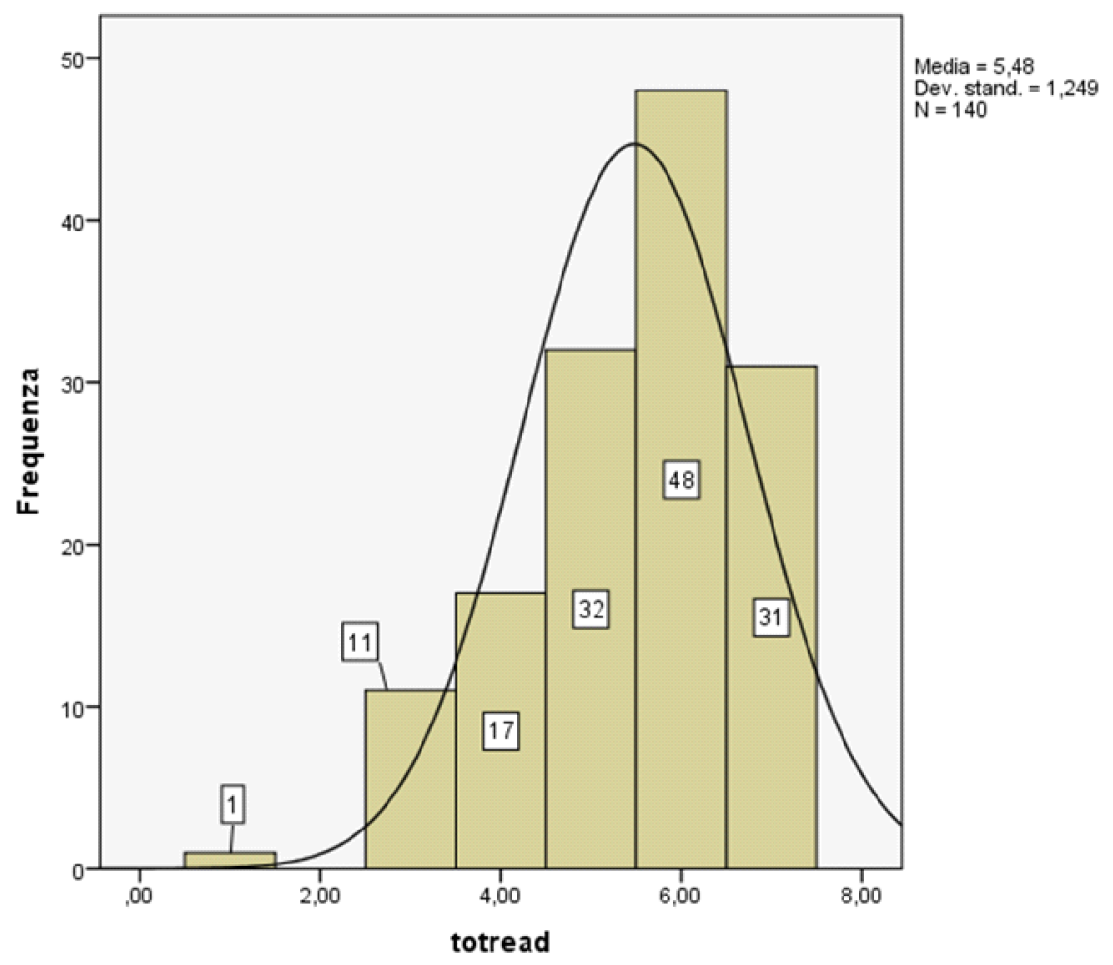

Fig. 5.Punteggi dei candidati alla prova di ascolto \# 1. Certificazione CILS, Sessione gingno 2012, livello B1

Consideriamo le specificità di ciascun item (riportiamo la trascrizione dei microdialoghi con evidenziata in grassetto la porzione di testo cui si riferisce la chiave). 
item \# 1

È un itemrelativamente facile. I distrattori non svolgono una funzione evidente.

- Salve, sono qui in vacanza con mia moglie. Avete una mappa della città?

- Si, ecco: in questa cartina trovate $i$ siti archeologici, $i$ monumenti e i musei principali di Roma.

- E per spostarci quale mezzo ci consiglia?

- I taxi a Roma non costano molto, ma vi conviene acquistare la carta Roma Pass: con 30 euro potete viaggiare gratis su autobus e metropolitana, e visitare tutti i monumenti e $i$ musei della città.

L'impiegata dell'agenzia turistica consiglia al turista di acquistare

A) un pacchetto di visite guidate per i musei.

B) una tessera giornaliera per la metropolitana.

C) un abbonamento settimanale al servizio di taxi.

D) una carta-sconto per monumenti e mezzi di trasporto.

\begin{tabular}{|c|c|c|c|c|c|}
\hline & & Frequenza & Percentuale & $\begin{array}{c}\text { Percentuale } \\
\text { valida }\end{array}$ & $\begin{array}{c}\text { Percentuale } \\
\text { cumulata }\end{array}$ \\
\hline \multirow{5}{*}{ Validi } & $\bar{A}$ & 8 & 5,7 & 5,8 & 5,8 \\
\hline & B & 6 & 4,3 & 4,4 & 10,2 \\
\hline & $\mathrm{C}$ & 5 & 3,6 & 3,6 & 13,9 \\
\hline & $\mathrm{D}=$ Chiave & 118 & 84,3 & 86,1 & 100,0 \\
\hline & Totale & 137 & 97,9 & 100,0 & \\
\hline Mancanti & Nessuna risposta & 3 & 2,1 & & \\
\hline Totale & & 140 & 100,0 & & \\
\hline
\end{tabular}

item \# 2

L'item pare discriminare adeguatamente.

Il distrattore c) attira, comunque, un discreto numero di consensi. Forse ciò è dovuto al fatto che la chiave non appare così evidente (non è menzionata, infatti, la città di partenza).

Può essere che coloro i quali hanno optato per il distrattore c) si siano affidati a una informazione fornita all'inizio del brano audio(il noleggio 
dell'auto per alcuni giorni), senza prestare attenzione ai dettagli (la durata del noleggio). A confermare tale ipotesi è anche il fatto che il $20 \%$ degli apprendenti che ha risposto correttamente (chiave a), ha scelto il distrattore c) a un primo ascolto, per poi ricorreggersi durante il secondo ascolto.

- Buongiorno, mi chiamo Ernesto Quintili, ho prenotato un'automobile on-line, una Panda, per tre giorni.

- Si, ha con sé la stampa della prenotazione?

- Eccola. Però vorrei riconsegnare l'auto a Messina, perché mi imbarco lì.

- Va bene, può riconsegnarla all'autonoleggio di Via Crispi. Penso io ad avvertirli.
2. Il signor Quintili vuole
A) riconsegnare l'auto in un'altra città.
B) pagare l'auto al termine del noleggio.
C) riconsegnare l'auto dopo due giorni.
D) cambiare l'auto che ha noleggiato.

\begin{tabular}{llrrrr}
\hline & & Frequenza & Percentuale & $\begin{array}{c}\text { Percentuale } \\
\text { valida }\end{array}$ & $\begin{array}{c}\text { Percentuale } \\
\text { cumulata }\end{array}$ \\
\hline \multirow{6}{*}{ Validi } & A=Chiave & 101 & 72,1 & 75,4 & 75,4 \\
& B & 9 & 6,4 & 6,7 & 82,1 \\
& $\mathrm{C}$ & 15 & 10,7 & 11,2 & 93,3 \\
& $\mathrm{D}$ & 9 & 6,4 & 6,7 & 100,0 \\
& Totale & 134 & 95,7 & 100,0 & \\
\multirow{5}{*}{ Mancanti } & Nessuna risposta & 5 & 3,6 & & \\
\multirow{2}{*}{ Totale } & Doppia risposta & 1 & 0,7 & & \\
& Totale & 6 & 4,3 & & \\
\hline
\end{tabular}

item \# 3

È l'item che presenta il FV minore.

- Paolo, hai fatto tu i biglietti per Ravenna?

- No, li facciamo domani in stazione: il treno parte alle 8. 
- Ma a quell'ora c'è sempre tanta gente alla biglietteria! Se perdiamo quel treno arriviamo tardi e non sappiamo neppure dove si trova il nostro albergo.

- Hai ragione, Laura, guardo su internet e prenoto i biglietti.

3. Laura vuole prenotare il treno delle ore 8 perché

A) teme di arrivare tardi a un appuntamento.

B) vuole evitare l'ora di punta del traffico.

C) ha paura di viaggiare di notte.

D) vuole avere il tempo per trovare l'albergo.

\begin{tabular}{llrrrr}
\hline & & Frequenza & Percentuale & $\begin{array}{c}\text { Percentuale } \\
\text { valida }\end{array}$ & $\begin{array}{c}\text { Percentuale } \\
\text { cumulata }\end{array}$ \\
\hline \multirow{4}{*}{ Validi } & A & 30 & 21,4 & 21,6 & 21,6 \\
& $\mathrm{~B}$ & 25 & 17,9 & 18,0 & 39,6 \\
& $\mathrm{D}=$ Chiave & 84 & 60,0 & 60,4 & 100,0 \\
Mancanti & Totale & 139 & 99,3 & 100,0 & \\
Totale & Nessuna risposta & 1 & 0,7 & & \\
\hline
\end{tabular}

Il ragionamento richiesto all'apprendente è molto arduo. Riproduciamo nello schema che segue il filo logico delle argomentazioni della donna (Laura), volte a far desistere l'uomo (Paolo) dall'acquistare i biglietti in stazione l'indomani. In neretto, i contenuti espliciti; l'ultima è una deduzione che l'ascoltatore può trarre. 


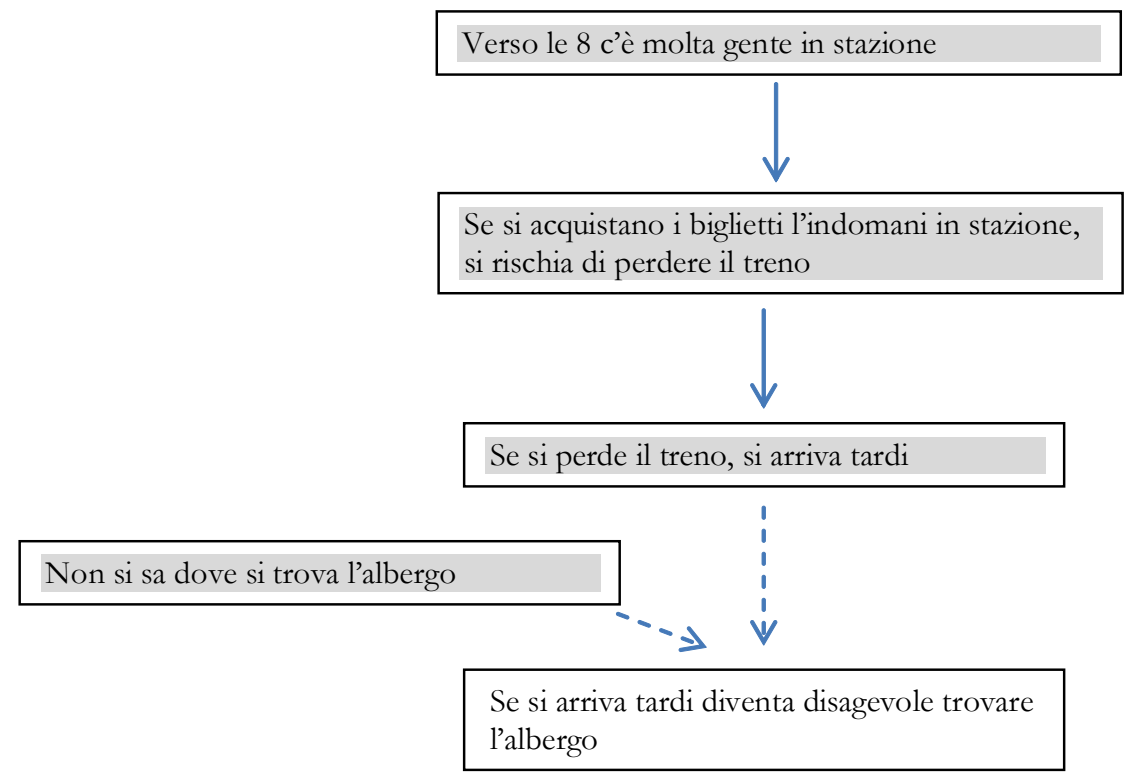

Fig. 6.Il ragionamento del personaggio femminile nell'audio corrispondente all'item \#3

La donna chiede all'uomo se ha provveduto ad acquistare i biglietti del treno per Ravenna. L'uomo risponde che li compreranno insieme l'indomani in stazione: conferma (o fa sapere) che prenderanno il treno delle otto (non sappiamo se di mattina o di sera).

Inizia l'argomentazione della donna: Laura sottolinea come sia complicato fare $\mathrm{i}$ biglietti in stazione a quell'ora (non sappiamo ancora a quale momento della gionnata si riferisca), dal momento che la stazione è affollata. Inoltre prefigura le conseguenze negative legate all'eventualità di non riuscire a fare i biglietti: dovrebbero prendere il treno successivo e arriverebbero "tardi" (non si capisce se l'avverbio si riferisca alle ultime ore della mattina o della sera, o se sia relativo a un appuntamento fissato). Aggiunge che sono ignari di dove si trovi il "loro"albergo (può essere che qualcuno li accolga alla stazione di arrivo e dica loro dove sia il "loro" albergo, confermando l'ipotesi dell'appuntamento, oppure può essere che debbano raggiungere l'albergo -a piedi presumibilmente- ma non conoscano molto 
bene Ravenna; tra l'altro, si potrebbe aggiungere, se il"tardi" equivale a notte inoltrata, potrebbero non trovare passanti che forniscano loro informazioni).

L'uomo riconosce le ragioni della donna e assicura che prenoterà $i$ biglietti in rete.

Ora, se consideriamo attentamente l'item riscontriamo problemi a vari livelli.

In primo luogo lo stem non è corretto. Laura vuole assicurarsi che riescano a prendere il treno delle otto ma è Paolo che prende l'iniziativa di prenotare (tra parentesi, la prenotazione non coincide con l'acquisto; quindi la coda la dovrebbero fare lo stesso il giorno seguente?); in effetti, in merito al problema sollevato da Laura, Paolo avrebbe potuto escogitare una soluzione diversa, come recarsi quel giorno stesso in stazionead acquistare $i$ biglietti.

In secondo luogo, idistrattori a) e b), scelti ciascuno da una percentuale considerevole del campione (circa un quinto), paionoavere una loro plausibilità. Per via di processi di inferenza, si può ritenere che:

- Laura e Paolo abbiano un appuntamento in agenda (distrattore a), a seguito del quale dovranno recarsi in albergo (potrebbero ricevere informazioni in merito all'albergo proprio durante l'incontro)

- il fatto di trovare "tanta gente alla biglietteria" può far presumere si tratti di un'ora di punta, con problemi di traffico relativo, e quindi con un affollamento in stazione (distrattore b).

In definitiva, riteniamo che l'item, poiché costruito su un reticolo argomentativo molto complesso, retto su una coordinazione di ipotesi,ricco di impliciti e con un'informazione non corretta nello stem, rischi di confondere il test taker, i risultati che ne ricaviamo non ci paiono attendibili.

item \# 4

È un item relativamente facile (FV 80,7\%). Il distrattore a) non è stato scelto da nessuno.

- Ciao Maria, sono Luca. Sei riuscita a trovare i biglietti per il concerto di Venditti? 
- Si, ne ho trovati due, viene anche la mia amica Vittoria.

- Anch'io ho i biglietti, sono con dei miei amici. Se vuoi, possiamo trovarci una mezz'ora prima del concerto per mangiare qualcosa insieme.

- Noi andiamo all'ultimo momento, perché usciamo tardi dal lavoro, ci vediamo al concerto.

4. Maria dice a Luca che

A) non ha trovato i biglietti del concerto.

B) va al concerto dopo il lavoro.

C) possono mangiare insieme dopo il concerto.

D) non può andare al concerto per motivi di lavoro.

\begin{tabular}{llrrrr}
\hline & Frequenza & Percentuale & $\begin{array}{c}\text { Percentuale } \\
\text { valida }\end{array}$ & $\begin{array}{c}\text { Percentuale } \\
\text { cumulata }\end{array}$ \\
\hline \multirow{4}{*}{ Validi } & B=Chiave & 113 & 80,7 & 82,5 & 82,5 \\
& $\mathrm{C}$ & 13 & 9,3 & 9,5 & 92,0 \\
& $\mathrm{D}$ & 11 & 7,9 & 8,0 & 100,0 \\
& Totale & 137 & 97,9 & 100,0 & \\
Mancanti & Doppia risposta & 3 & 2,1 & & \\
Totale & & 140 & 100,0 & & \\
\hline
\end{tabular}

item \# 5

Si tratta di un item facile. Solo il distrattore d) risulta insidioso; in effetti la porzione di testo a cui l'itemsi riferisce, "Vorrei qualcosa che posso trasportare in aereo. Parto domani e il volo dura 14 ore", ammette due inferenze: il trasporto in aereo implica un problema di spazio o di conservazione del cibo? È il dettaglio del tempo di volo ("14 ore") a far piegar la scelta verso la seconda opzione, la chiave appunto, e quindi a essere decisivo.

- Buongiorno, vorrei comprare dei prodotti tipici della Puglia.

- Si, che cosa preferisce? Abbiamo la pasta fresca oppure il pane di Altamura o iformaggi.

- Veramente vorrei qualcosa che posso trasportare in aereo. Parto domani e il volo dura 14 ore.

- Allora può prendere solo la pasta. 


\section{La signora vuole acquistare cibi che}

A) si conservano durante il viaggio.

B) contengono ingredienti naturali.

C) hanno una data di scadenza lunga.

D) occupano poco spazio in valigia.

\begin{tabular}{llrrrr}
\hline & Frequenza & Percentuale & $\begin{array}{c}\text { Percentuale } \\
\text { valida }\end{array}$ & $\begin{array}{c}\text { Percentuale } \\
\text { cumulata }\end{array}$ \\
\hline \multirow{6}{*}{ Validi } & A=Chiave & 119 & 85,0 & 85,6 & 85,6 \\
& $\mathrm{~B}$ & 3 & 2,1 & 2,2 & 87,8 \\
& $\mathrm{C}$ & 3 & 2,1 & 2,2 & 89,9 \\
& $\mathrm{D}$ & 14 & 10,0 & 10,1 & 100,0 \\
Mancanti & Totale & 139 & 99,3 & 100,0 & \\
Totale & Nessuna risposta & 1 & 0,7 & & \\
\hline
\end{tabular}

item \# 6

L'item è relativamente facile, con un discreto potere attrattivo dell'ultimo distrattore $(11,4 \%)$, legato forse al fatto che il cameriere parla di "piatti di verdure" (anche se non specificatamente di "antipasto" né di "verdure crude").

- Siete pronti, volete ordinare?

- Si, grazie. Per mio figlio un piatto di spaghetti al pomodoro e una bistecca, io vorrei un'insalata mista e un piatto di formaggi.

- Va bene. Se vuole le porto il menù vegetariano: abbiamo molti piatti di verdure.

- La ringrazio, è molto gentile.

6. Il cameriere dice alla signora che il ristorante

A) ha finito le verdure.

B) ha un nuovo cuoco.

C) offre molte ricette vegetariane.

D) propone un antipasto di verdure crude. 


\begin{tabular}{llrrrr}
\hline & & Frequenza & Percentuale & $\begin{array}{c}\text { Percentuale } \\
\text { valida }\end{array}$ & $\begin{array}{c}\text { Percentuale } \\
\text { cumulata }\end{array}$ \\
\hline \multirow{5}{*}{ Validi } & A & 4 & 2,9 & 2,9 & 2,9 \\
& B & 1 & 0,7 &, 7 & 3,6 \\
& C =Chiave & 118 & 84,3 & 84,9 & 88,5 \\
& D & 16 & 11,4 & 11,5 & 100,0 \\
Mancanti & Totale & 139 & 99,3 & 100,0 & \\
Totale & Doppia risposta & 1 & 0,7 & & \\
\hline
\end{tabular}

item \# 7

L'item è relativamente facile. Il distrattore c) attrae comunque un discreto numero di consensi: non è chiaro se, per coloro che l'hanno scelto, l'andare a vedere "la mostra sul Divisionismo" in un'altra città (Rovigo) possa essere inteso come una "gita" (posto che la città sia relativamente distante da quella in cui abitano gli interlocutori).

- Ciao Marina, ti chiamo perché domenica prossima io e Andrea andiamo alla mostra sul Divisionismo a Rovigo. Ti interessa?

- Verrei volentieri, ma domenica si sposa mia nipote. Non possiamo rimandare tra 15 giorni?

- Purtroppo no, domenica 24 è l'ultimo giorno della mostra.

- Peccato, mi dispiace molto.
7. Marco telefona a Marina per invitarla
A) a visitare una mostra.
B) al matrimonio di sua nipote.
C) a una gita a Rovigo.
D) a sentire un concerto.

\begin{tabular}{llrrrr}
\hline & Frequenza & Percentuale & $\begin{array}{c}\text { Percentuale } \\
\text { valida }\end{array}$ & $\begin{array}{c}\text { Percentuale } \\
\text { cumulata }\end{array}$ \\
\hline \multirow{6}{*}{ Validi } & A=Chiave & 114 & 81,4 & 83,2 & 83,2 \\
& $\mathrm{~B}$ & 6 & 4,3 & 4,4 & 87,6 \\
& $\mathrm{C}$ & 16 & 11,4 & 11,7 & 99,3 \\
& $\mathrm{D}$ & 1 & 0,7 & 0,7 & 100,0 \\
Mancanti & Totale & 137 & 97,9 & 100,0 & \\
Totale & Nessuna risposta & 3 & 2,1 & & \\
\hline
\end{tabular}




\subsubsection{Bilancio}

Come già detto, l'esercizio si presenta maggiormente calibrato rispetto agli altri due. Si rileva, comunque, la presenza di un itemproblematico (\# 3), sia nella struttura del testo audio (il quale è tutt'altro che "lineare", come vorrebbe invece il Quadro di Riferimento per il livello B1) che per come si presentano stem e opzioni.

\section{Conclusione}

Questa nostra indagine ci consente di ragionare sul comportamento degliitem di una prova chiusa. Emerge, nel caso specifico, una relativa facilità delle prove, con la necessità di ricalibrare vari item sottodimensionati. A livello di design siamo convinti sia necessario disambiguare alcune opzioni e prestare attenzione a che la velocità di eloquio risulti adeguata al livello e in ogni caso, per quanto possibile, non contraddistinta da pause innaturali. Da ultimo, per quanto concerne i testi estesi, si raccomanda una corrispondenza lineare tra item e informazioni presenti nell'audio e una distribuzione adeguata degli item.

\section{Riferimenti bibliografici}

BACHMAN, L. F. Fundamental considerations in language testing. Oxford: OUP, 1990.

COUNCIL OF EUROPE. The Common European Framework of reference for languages: learning, teaching, assessment. Cambridge: CUP, 2001. (Edizione italiana: Quadro comune europeo di riferimento per le lingue: apprendimento, insegnamento, valutazione. Firenze: La Nuova Italia).

FEHÉRVÁRYNÉ, K.; PIŽORN, K. Listening. Budapest: British Council-Teleki Foundation, 2005.

GREEN, R. Statistical analyses for language teachers. Basingtoke: Palgrave MacMillan, 2013.

McNAMARA, T. Language testing. Oxford: OUP, 2000. 
NOVELLO, A. Testare l'ascolto: l'abilità e linee-guida per la costruzione di item. Officina. it, 22, 2014. <http://bit.do/bG2BA>. Ultimo accesso: 14 ott. 2015.

POPHAMJ, W. Modern educational measurement. Boston: Allyn \& Bacon, 2000.

\section{Riferimenti sitografici}

INTO EUROPE PROJECT, British Council, Hungary.

<http://bit.do/bG2BY>. Ultimo accesso: 14 ott. 2015.

CERTIFICAZIONE CILS, Prove B1, sessione Giugno 2009.

<http://bit.do/bG2CF>. Ultimo accesso: 14 ott. 2015.

CERTIFICAZIONE CILS, Prove B1, sessione Giugno 2012.

<http://bit.do/bG2CN>. Ultimo accesso: 14 ott. 2015.

Recebido em: 19/11/2014

Aceito: 02/04/2015 